\title{
Non-destructive, position-selective, and multi-elemental analysis method involving negative muons
}

\author{
Kazuhiko NINOMIYA* \\ Graduate School of Science, Osaka University, 1-1 Machikaneyama, Toyonaka, Osaka 560-0043, Japan
}

Received July 14, 2019; Accepted September 27, 2019; Published online October 4, 2019

\begin{abstract}
Elemental analysis is of fundamental importance in several scientific domains. Many elemental analysis methods have been developed till date, with particular importance having been given to non-destructive analysis methods. In this paper, we review a novel non-destructive, position-selective, and multi-elemental analysis method for bulk material that utilizes a new type of probe, a negative muon. When a muon is stopped by an atom in a material, muonic X-rays are emitted. Due to the large mass of a muon, the energy of muonic X-rays is very high, and the "X-ray fluorescence analysis" method using a muon can solve the problem of self-absorption in an ordinary method using an electron. Two studies involving quantitative and depth-profiling analysis of archeological artifacts by this method are reviewed in this work. We also discuss the scope for future research using this method.
\end{abstract}

\section{Introduction}

Many elemental analysis methods that utilize various probes have been developed till date in several research fields. Among them, non-destructive analysis methods are particularly useful, although these have proven to have accuracies inferior to destructive analysis methods in most cases. The usefulness of non-destructive methods stems from the fact that a sample can be utilized for further processes after a round of non-destructive analysis. Therefore, non-destructive methods are advantageous for the analysis of valuable samples. The development of an elemental analysis method that enables the analysis of all elements in a given sample, even bulk samples with high positional sensitivity, is a major goal for analytical scientists at present. Recently, a new elemental analysis method that satisfies these conditions by utilizing a negative muon (MIXE: muon induced x-ray emission) has been developed and applied in several studies ${ }^{1-6}$. A negative muon is an elementary particle, and intense muon beams generated in an accelerator are used in many studies. With recent advancement in accelerator technology to produce intense muon beams, it has become possible to use muons for practical elemental analyses ${ }^{7-10}$.

A muon's mass is about 207 times that of an electron $\left(105.658 \mathrm{MeV} / \mathrm{c}^{2}\right)$. When a muon is injected into a substance, it gradually loses its kinetic energy due to its interaction with electrons and finally comes to a stop. Because a negative muon has the same charge as an electron, it binds with nuclei. An atom in which a negative muon has replaced an electron is called a muonic atom. The binding energies of a muon in the orbital states of a muonic atom are about 200 times higher than those of an electron with the corresponding principal quantum number. Although much research has been conducted on the muon capture process in an atom ${ }^{11-13}$, the process is still not completely understood. During the initial stages of muonic atom formation, the captured muons are highly excited. However, then, because there is only one muon in a muonic atom, and all other muon atomic orbits are vacant, the muon immediately de-excites to the muonic 1s state. As a result, electrons by muon-electron Auger processes and characteristic $\mathrm{X}$-rays (muonic X-rays) are emitted. Since a muon is 200

*Corresponding author. E-mail: ninokazu@chem.sci.osaka-u.ac.jp Fax: +81-6-6850-6999* times heavier than an electron, the muon atomic orbit is much closer to the nucleus than the electron atomic orbit and the binding energy is much larger. Therefore, the energies of muonic X-rays are 200 times higher than those of the characteristic X-rays of electrons. After a muon reaches the 1s muon atomic orbit, it either decays into an electron and two neutrinos within its lifetime ${ }^{14}$, or gets absorbed into the nucleus in the case of heavy nuclei. The muon absorption reaction is very similar to EC (electron capture) decay, leading to the formation of a Z-1 nucleus ${ }^{15}$.

Muonic X-rays can be applied to elemental analysis by $\mathrm{X}$-ray spectroscopy as well as X-ray fluorescence. As aforementioned, because muonic X-rays have higher energy than the characteristic X-rays from electrons, muonic X-rays can penetrate samples easily. As a result, the impact of X-ray absorption by the sample on the results can be ignored in a muonic X-ray measurement; this is a significantly large problem in X-ray fluorescence analysis. Further, even light elements such as carbon, that are difficult to analyze using conventional X-ray fluorescence analysis have large muonic $\mathrm{X}$-ray energies. For example, the energy of the $\mathrm{K}_{\alpha} \mathrm{X}$-ray (2p1s transition) of a muonic carbon atom is $75 \mathrm{keV}^{16}$, which is the energy corresponding to the lead KX-ray line in the case of ordinary X-ray fluorescence. The probability of muonic X-ray emission depends on the probability of muonic atom formation. One muonic atom is formed when a muon stops in it, and multiple muonic X-rays are emitted during the muon de-excitation process ${ }^{17}$. Therefore, highly sensitive elemental analysis is possible using muonic X-rays. However, it is difficult to predict muon capture probabilities in each element precisely because the probabilities are slightly altered by the chemical environment of the muon capturing atom ${ }^{18-20}$. It was revealed in our previous work ${ }^{1}$ that the muon capture probability for an element is almost independent of the element concentration (in wt \%) in a sample, implying that the analytical sensitivity is hardly be altered. When a muon beam is generated by an accelerator, the incident energy into the sample can be selected by the magnetic system in the beam transportation line. Since the muon stopping depth in a sample depends on the incidence energy of a muon, it is possible to control the position of muonic X-ray emissions, allowing the position of analysis to be selected $d^{2,3,21}$. For these reasons, we agree that muonic X-ray measurement has superior features for elemental analysis: its 


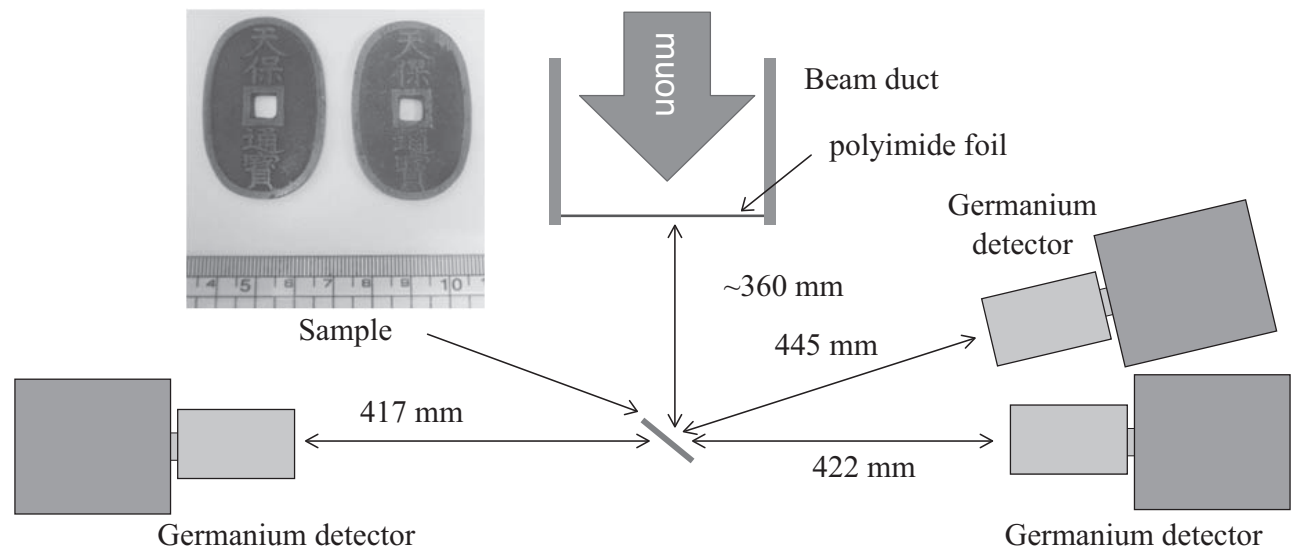

Figure 1. Schematic view of the experimental setup and photograph of the samples. The muon beam entered the air layer and irradiated on the sample. The sample was set at $45^{\circ}$ with the beam direction. Muonic X-rays were measured by three high-purity germanium detectors. Two Tempo-Tsuho coins were subjected to the muon-irradiation experiment; the left one is authentic, called Edo-Seiji, and the right one is an imitation, called Mito-Seiji.

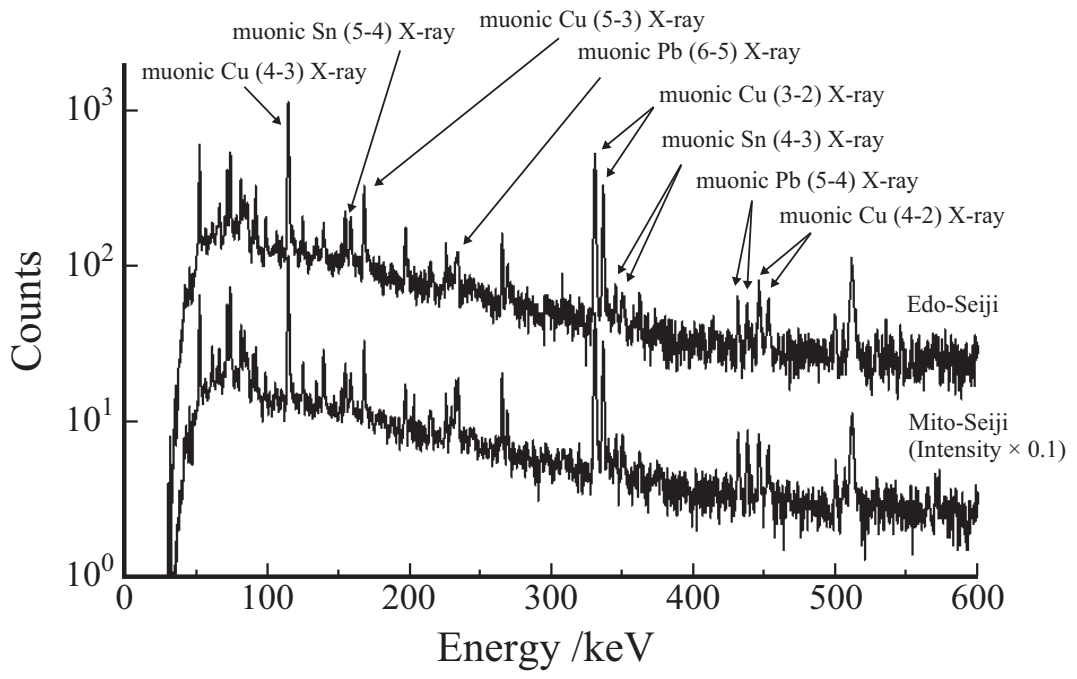

Figure 2. Muonic X-ray spectrum for two Tempo-Tsuho coins: Edo-Seiji and Mito-Seiji. Muonic X-rays originating from muon capture on Cu, $\mathrm{Sn}$, and $\mathrm{Pb}$ are clearly observed. The numbers in parentheses indicate the change of the principal quantum number with the transition of the atomic muon.

use makes the method non-destructive, multi-elemental, including light elements, and position selective.

The possibility of element analysis using muons was pointed out over 40 years ago ${ }^{22}$, following which some pioneering studies have been conducted in this field ${ }^{23-26}$. However, previous works largely performed qualitative analyses due to a lack of understanding of the muonic atom formation process and difficulty in obtaining good quality data from low-intensity muon beams. In recent years, fundamental research on muonic atom formation has been performed using an intense muon beam source in the Japan Proton Accelerator Research Complex (J-PARC) ${ }^{27-31}$. The use of such intense muon beam sources has made elemental analysis using muonic X-rays increasingly possible and significant progress has been made in applying this method to various samples, such as archeological artifacts, extraterrestrial materials, etc. ${ }^{1-3,32,33}$. In this paper, we review two elemental analysis methods for archeological material, which were developed and verified at J-PARC.

\section{Quantitative elemental analysis of the interior of a bronze artifact}

To determine the elemental composition of old bronze coins non-destructively ${ }^{1}$, muonic X-ray measurements were performed in the D2 experimental area of the MUon Science
Establishment (MUSE) in the Materials and Life Science Facility (MLF), the Japan Proton Accelerator Research Complex (J-PARC) ${ }^{9}$. The schematic of the experimental setup is given in Figure 1. The muon beam from the $\mathrm{D} 2$ beamline was passed through a polyimide foil, then air, and finally irradiated onto the sample. Three coaxial-type germanium semiconductor detectors (GMX20, ORTEC Inc. USA) were placed around the sample to measure the muonic X-rays emitted from the muonic atoms generated after the muon stopped in the sample. To reduce the background events, the measurement of the muonic X-rays was performed only at the times coincident with muon beam pulses. The muon irradiation was conducted for each bronze coin over approximately $10 \mathrm{~h}$. These bronze coins were similar in their appearance, as shown in Figure 1, but were made in different places. The coin on the left (EdoSeiji) in Figure 1 is authentic while the one on the right (MitoSeiji) is an imitation with different elemental compositions. Muon irradiation analyses were also conducted for standard bronze samples whose elemental compositions were already determined by ICP-AES (Inductively Coupled Plasma Atomic Emission Spectroscopy) were also conducted. The energy of the incident muon was $35 \mathrm{MeV} / \mathrm{c}$, which corresponds to a stopping depth of $\sim 250 \mu \mathrm{m}$ from the sample surface. The spread in the distribution of the muon stopping depth was estimated to be $10 \%$ at the stopping position, about $25 \mu \mathrm{m}$ in this case.

Figure 2 shows the spectra obtained by muon irradiation of 

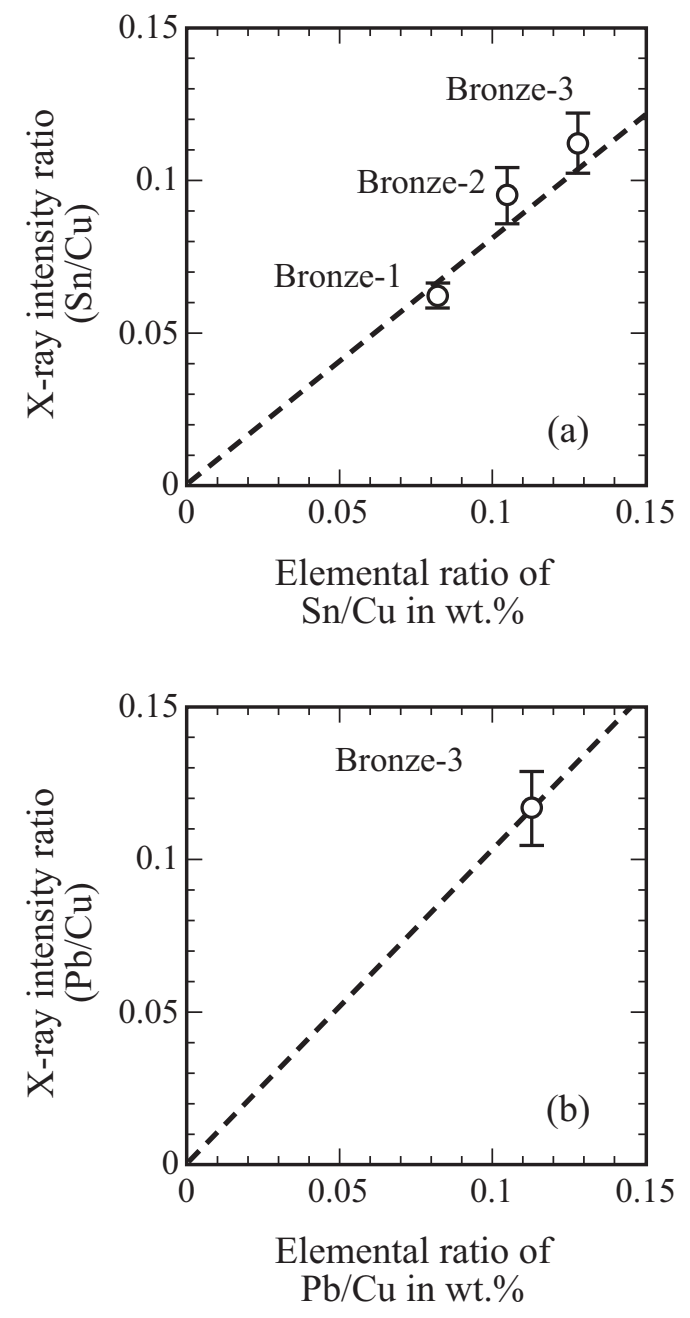

Figure 3. The relationship between the elemental ratios in weight percent and muonic X-ray intensity ratios for (a) $\mathrm{Sn} / \mathrm{Cu}$ and (b) $\mathrm{Pb} /$ $\mathrm{Cu}$.

for the bronze coins. The muonic X-rays from copper, tin, and lead atoms, which are the constituent elements of bronze, could be clearly observed. The muonic X-ray spectra of the bronze coins are very similar to each other, but have different intensities in each line. Since the intensity of the muonic $\mathrm{X}$-rays correspond to the number of muons captured in each element, it is possible to determine the elemental composition from the intensity of the muonic X-rays. By focusing on the muonic X-ray intensities for muonic copper (4-3), muonic tin (5-4) and muonic lead (6-5), which have the highest counting statistics in the measured energy range for each element, the elemental compositions of the bronze coins were quantitatively determined. The numbers in parentheses indicate the change in the principal quantum number with the transition of the muonic atom; for example, (4-3) implies muonic $\mathrm{N}_{\alpha} \mathrm{X}$-ray.

Figure 3 shows the relationship between the elemental compositions and muonic X-ray intensities of $\mathrm{Sn} / \mathrm{Cu}$ and $\mathrm{Pb} / \mathrm{Cu}$ obtained via measurements of the standard bronze samples. As shown in Figure 3(a), the X-ray intensity of the muonic tin atom increases with increase in the elemental composition of tin in the sample. The linear relationship between the muonic $\mathrm{X}$-ray intensities (muon capture probabilities) and elemental composition with various alloys ${ }^{34}$, can be determined from the calibration line, as shown in Figure 3. Additionally, the slope of the proportional relationship is approximately 1 . The linearity implies that the relative weight percentages of these metals mostly correspond to the muonic X-ray intensity ratios; that is, the sensitivity is almost the same for any element, as discussed above ${ }^{1}$. From the muonic X-ray intensity of the bronze coin, the elemental compositions were determined

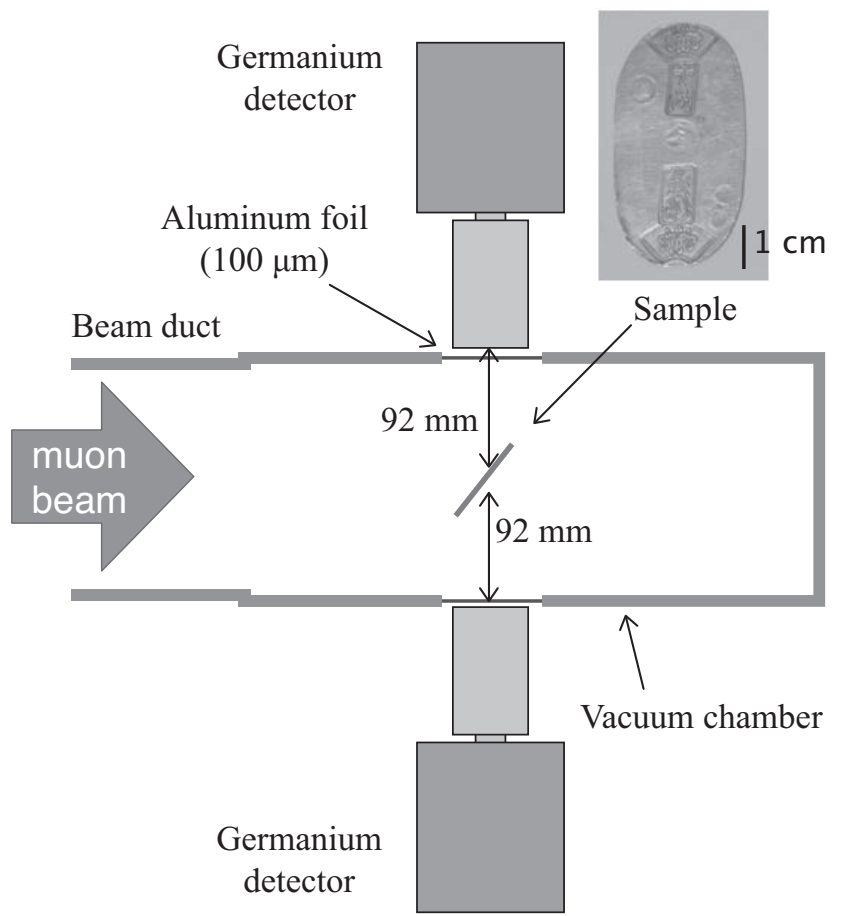

Figure 4. Experimental setup for elemental depth profiling for the Tempo-koban sample with $0.2,0.27,0.30,0.49$, and $1.0 \mathrm{MeV}$ incident muon energy conditions. The irradiations were conducted in the vacuum chamber. Muonic X-rays were measured by two germanium detectors. The sample was set at $45^{\circ}$ with the muon beam direction. The experiment with $5.6 \mathrm{MeV}$ irradiation condition was performed without the vacuum chamber as described in a previous paper ${ }^{35}$.

using the relationship shown in Figure 3. They were found to be $\mathrm{Cu}: 77.7 \pm 1.6 \%$, Sn: $12.5 \pm 1.5 \%$, and $\mathrm{Pb}: 9.8 \pm 1.5 \%$ for the Edo-Sheiji sample and $\mathrm{Cu}: 69.0 \pm 1.9 \%$, Sn: $9.9 \pm 1.3 \%$, and $\mathrm{Pb}$ : $21.1 \pm 2.6 \%$ for the Mito-Seiji sample. This indicates that though two bronze coins seem to have the same appearance, they have different elemental compositions. These results are consistent with the results of surface elemental analysis using X-ray florescence; $\mathrm{Cu}: 77.8 \%, \mathrm{Sn}: 14.5 \%$, and $\mathrm{Pb}: 7.8 \%$ for the Edo-Seiji sample and $\mathrm{Cu}: 73.6 \%$, Sn: $12.2 \%$, and $\mathrm{Pb}: 14.2 \%$ for the Mito-Seiji sample. It should be noted that conventional $\mathrm{X}$-ray fluorescence analysis determines only the surface elemental composition, while muonic X-ray analysis identifies the deep interior of the sample. Thus, the bulk elemental composition of the bronze coins was successfully quantitatively determined without destroying the samples.

\section{Non-destructive depth-profiling of gold-silver alloy}

The stopping depth of an incident muon can be controlled by adjusting the current of the beamline magnets and selecting the incident muon energy. Non-destructive elemental depthprofiling analysis was carried out by changing the incident muon energy for an old Japanese gold coin of Tempo-koban, made in the nineteenth century ${ }^{3,35}$. The bulk gold component of the Tempo-koban is about $57 \mathrm{wt} \%$, and most of its residue is silver $^{36}$. In addition, the Tempo-koban has a gold rich layer near the surface that is several micrometers thick owing to chemical treatment to enhance the gold brilliance ${ }^{36}$. The experiment was conducted at the MUSE D2 beamline in the MLF J-PARC. The incident energies of muon were $0.20,0.27$, $0.30,0.49,1.0$, and $5.6 \mathrm{MeV}$; the stopping depths of these incident muon energies in the Tempo-koban was calculated to be $1.6,2.2,2.4,4.7,15.0$, and $210 \mu \mathrm{m}$, respectively. The details of the calculation procedures are described in our previous report $^{3}$. Figure 4 shows the schematics of the experimental setup. Unlike the experiment with bronze coins, in this case 


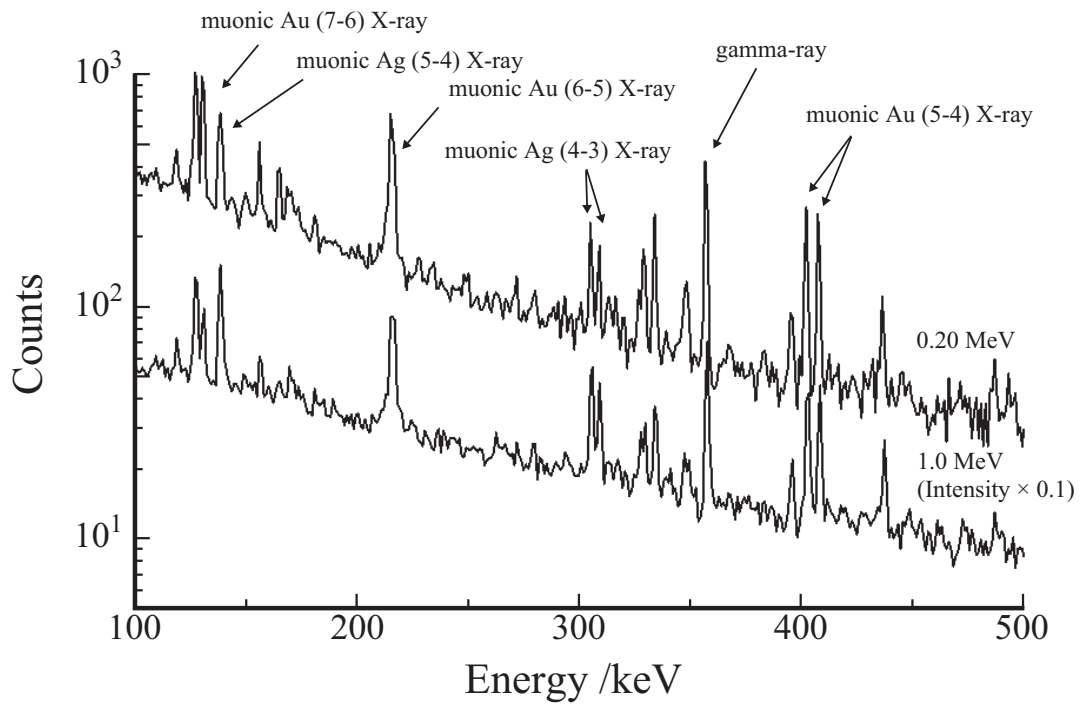

Figure 5. Muonic X-ray spectra for the Tempo-koban with 0.20 and $1.0 \mathrm{MeV}$ incident muon energies. Muonic X-rays of gold (5-4) and silver (4-3) were clearly identified. The X-ray intensity ratios of $\mathrm{Au} / \mathrm{Ag}$ were found to be clearly different based on the incident muon energies.

the muon irradiation was performed in vacuum, except at 5.6 $\mathrm{MeV}$, because a low-energy muon is stopped by the air layer before reaching the sample. The muonic X-rays emitted from the sample were measured using HPGe detectors. Muon irradiations on standard gold-silver alloys containing 50, 60, and $80 \mathrm{wt} \%$ of gold were also performed.

Figure 5 shows the muonic X-ray spectra in the 100-500 $\mathrm{keV}$ region for the Tempo-koban sample with two incident muon energies 0.20 and $1.0 \mathrm{MeV}$. The muonic X-rays originating from the muon captures by the gold (muonic gold (5-4) line) and silver (muonic silver (4-3) line) atoms can be clearly identified. The intensity ratios of the X-rays emitted from the gold and silver atoms appear to be dependent on the incident muon energies, implying that the elemental compositions of the Tempo-koban are different owing to different stopping depths of the muon. To conduct quantitative analysis, the calibration curve between the muonic X-ray intensity and the elemental composition ratio was obtained in the same manner as that in the experiment with bronze: by analyzing the data of the standard samples of $\mathrm{Au} / \mathrm{Ag}$ alloy, and obtaining a linear relationship (see Figure 6). From this relationship and the intensity of the muonic X-ray, the gold content corresponding to each muon stopping depth was determined, as shown in Figure 7. The content of gold was about $80 \%$ near the surface and about $57 \%$ at depths greater than $2.4 \mu \mathrm{m}$ from the surface in Tempo-koban. The results for both the surface and interior of the Tempo-koban were consistent with the values determined by destructive analysis. Thus, using this method, accurate elemental depth-profiling is possible by muon irradiation using various muon incident energies.

\section{Isotopic analysis by muonic KX-ray measurement}

Since the muon atomic orbitals are very close to the nucleus, the energies of these levels are strongly influenced by the proton distribution of the nucleus, i.e., isotope ${ }^{37}$. As a result, a large isotopic shift on the muonic X-ray is observed, particularly for KX-rays, which involve the orbit closest to the nucleus during the transition. Proton distributions have been studied for various nuclei by measuring the muonic KX-ray energies $^{38,39}$. In other words, it is possible to identify the isotopes by muonic X-ray measurements; thus, in addition to elemental analysis, isotopic analysis is also possible using the muonic $\mathrm{X}$-ray measurement owing to the isotopic shift of the muonic $\mathrm{X}$-rays. In a recent work, non-destructive isotopic analysis by the muonic X-ray measurement was conducted for lead sam-

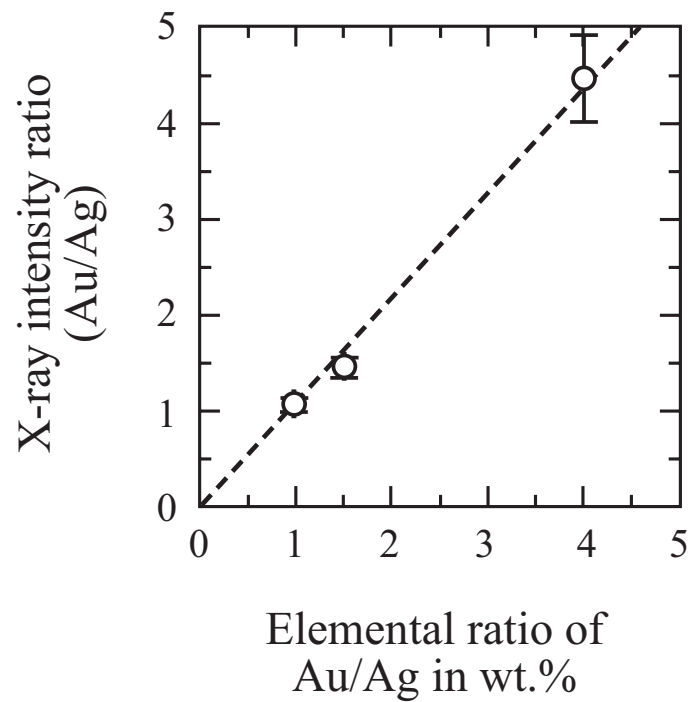

Figure 6. The relationship between the elemental ratio of $\mathrm{Au} / \mathrm{Ag}$ in weight percent and X-ray intensity ratio obtained from muon irradiations for gold-silver alloys with gold contents of 50, 60, and $80 \mathrm{wt} \%$.

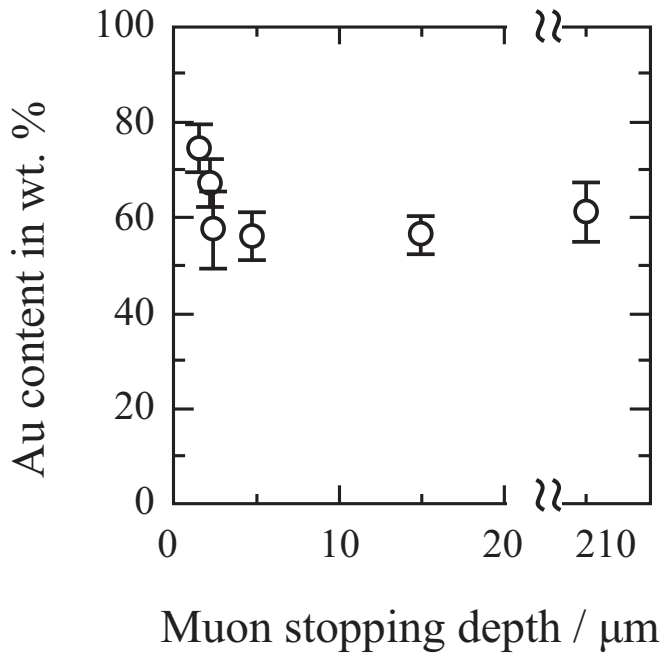

Figure 7. Elemental depth profiling of the Tempo-koban in gold contents. The gold concentration near the surface is higher than that in deep regions. This depth profile is consistent with the analysis results from the destructive approach ${ }^{36}$. 
ples for the first time, and the results were consistent with those from mass spectrometry ${ }^{40}$. Owing to the absence of practical methods for non-destructive isotopic analysis, a muon-based method has potential for application in various scientific studies.

\section{Three-dimensional elemental analysis}

The elemental depth-profiling analysis by the muon has already been achieved, as discussed using the example of Tempo-koban. In addition, further attempts to develop threedimensional (3D) elemental analyses have already been made by combining the depth information from an incident muon energy with two-dimensional muonic X-ray detectors ${ }^{41,42}$. A large solid-angle CdTe imaging detector was developed for a space observation satellite ${ }^{43}$. This detector was used by Katsuragawa et al. in 3D imaging analysis with muons in muon irradiation experiments for a patterned LiF sample sealed within an aluminum box. By controlling the incident muon energies, the muons were selectively stopped in LiF inside the aluminum box, and the muonic X-rays of fluorine atoms were measured. The pattern of LiF in the aluminum box was clearly identified with a spherical resolution of a few hundred microns. By further improving the detectors, it is expected that clearer images can be obtained, and 3D elemental analysis by muons can be developed.

\section{Measurement of gamma rays emitted after nuclear cap- ture of muon}

Similar to muonic X-rays, the gamma rays emitted after the formation of muonic atoms can also be used for the elemental analysis. After reaching the 1s orbit, the muon is absorbed by the nucleus through weak interactions. By this reaction, one proton in the nucleus is converted to one neutron in the nucleus and a Z-1 nucleus is formed. Because the mass of the muon is converted to the excitation energy of the nucleus, the resulting Z-1 nucleus is in a highly excited state. A part of this excitation energy is removed by the neutrino generated in the muon absorption reaction. From the excited Z-1 nucleus, the gamma rays and/or neutrons are emitted in the de-excitation process ${ }^{44}$. If the residual Z-1 nucleus is unstable, it further leads to gamma-ray emission and decay. The studies on the muon nuclear absorption processes and nuclear structures have employed gamma-ray measurements ${ }^{45,46}$. In addition to the elemental analysis ${ }^{47}$, isotopic analyse ${ }^{48}$ have also been conducted by gamma-ray measurements, with the results being consistent with the values determined by other methods, including muonic X-ray measurements.

\section{Summary and future scope}

In recent years, owing to the availability of high-intensity muon beams, non-destructive elemental analysis methods using muons have been developed and applied to samples in various research fields. Recently, non-destructive isotopic analysis for heavy elements and 3D analysis have also become possible. Further development is expected in various directions, for example, development of high-resolution 3D elemental analysis by improving the imaging detector, high-precision analysis by developing measurement systems with large solid angles and high efficiency using multiple germanium detectors, and non-destructive isotopic analysis including light elements using high-energy resolution detectors. In the future, this method of elemental analysis can be applied to diverse scientific disciplines such as archeology and geology.

\section{References}

(1) K. Ninomiya, T. Nagatomo, K. Kubo, T. U. Ito, W. Higemoto, M. Kita, A. Shinohara, P. Strasser, N. Kawamura, K. Shimomura, Y. Miyake, T. Saito, Bull. Chem. Soc. Jpn. 85, 228 (2012).

(2) K. Terada, K. Ninomiya, T. Osawa, S. Tachibana, Y. Miyake, M. K. Kubo, N. Kawamura, W. Higemoto, A. Tsuchiyama, M. Ebihara, M. Uesugi, Sci. Rep. 4, 5072 (2014).

(3) K. Ninomiya, M. K. Kubo, T. Nagatomo, W. Higemoto, T. U. Ito, N. Kawamura, P. Strasser, K. Shimomura, Y. Miyake, T. Suzuki, Y. Kobayashi, S. Sakamoto, A. Shinohara, T. Saito, Anal. Chem. 87, 4597 (2015).

(4) M. K. Kubo, J. Phys. Soc. Jpn. 85, 091015 (2016).

(5) A. D. Hillier, D. McK. Paul, K. Ishida, Microchem. J. 125, 203 (2016).

(6) K. Terada, A. Sato, K. Ninomiya, Y. Kawashima, K. Shimomura, G. Yoshida, Y. Kawai, T. Osawa, S. Tachibana, Sci. Rep. 7, 15478 (2017).

(7) E. Morenzoni, F. Kottmann, D. Maden, B. Matthias, M. Meyberg, T. Prokscha, T. Wutzke, U. Zimmermann, Phys. Rev. Lett. 72, 2793 (1994).

(8) T. Matsuzaki, K. Ishida, K. Nagamine, I. Watanabe, G. H. Eaton, W. G. William, Nucl. Instrum. Meth. Phys. Res. A 465, 365 (2001).

(9) W. Higemoto, R. Kadono, N. Kawamura, A. Koda, K. M. Kojima, S. Makimura, S. Matoba, Y. Miyake, K. Shimomura, P. Strasser, Quantum Beam Sci. 1, 11 (2017).

(10) S. Cook, R. D'Arcy, A. Edmonds, M. Fukuda, K. Hatanaka, Y. Hino, Y. Kuno, M. Lancaster, Y. Mori, T. Ogitsu, H. Sakamoto, A. Sato, N. H. Tran, N. M. Truong, M. Wing, A. Yamamoto, M. Yoshida, Phys. Rev. Accel. Beams. 20, 030101 (2017).

(11) L. I. Ponomarev, Ann. Rev. Nucl. Sci. 23, 395 (1973).

(12) A. Shinohara, T. Muroyama, C. Murata, T. Miura, T. Saito, A. Yokoyama, S. Kojima, M. Furukawa, Phys. Rev. Lett. 76, 2460 (1996).

(13) K. Kirch, D. Abbott, B. Bach, P. Hauser, P. Indelicato, K. Kottmann, J. Missimer, P. Patte, R. T. Siegel, L. M. Simons and D. Viel, Phys. Rev. A 59, 3375 (1999).

(14) T. Suzuki, D. F. Measday, J. P. Roalsvig, Phys. Rev. C 35, 2212 (1987).

(15)D. F. Measday, Phys. Rep. 354, 243 (2001).

(16) R. Engfer, H. Schneuwly, J. L. Vuilleumier, H. K. Walter, A. Zehnder, Atom. Data Nucl. Data Tab. 14, 509 (1974).

(17) P. Vogel, Phys. Rev. A 22, 1600 (1980).

(18) H. Daniel, Phys. Rev. Lett. 35, 1649 (1975).

(19) H. Schneuwly, V. I. Pokrovsky, L. I. Ponomarev, Nucl. Phys. A312, 419 (1978).

(20) N. Imanishi, S. Miyamoto, Y. Takeuchi, A. Shinohara, H. Kaji, Y. Yoshimura, Phys. Rev. A 37, 43 (1988).

(21) B. Hampshire, K. Butcher, K. Ishida, G. Green, D. Paul, A. Hillier, Heritage 2, 400 (2019).

(22) H. Daniel, Nuclear-Medizin 8, 311 (1969).

(23)E. Köhler, R. Bergmann, H. Daniel, P. Ehrhart, F. J. Hartmann, Nucl. Instrum. Meth. Phys. Res. 187, 563 (1981).

(24) H. Daniel, F. J. Hartmann, E. Köhler, Fresenius Z. Anal. Chem. 321, 65 (1985).

(25) Y. Hosoi, T. Watanabe, R. Sugita, Y. Tanaka, K. Nagamine, T. Ono, K. Sakamoto, British J. Radiology 68, 1325 (1995).

(26) M. K. Kubo, H. Moriyama, Y. Tsuruoka, S. Sakamoto, E. Koseto, T. Saito, K. Nishiyama, J. Radioanal. Nucl. Chem. 278, 777 (2008).

(27) K. Ninomiya, T. U. Ito, W. Higemoto, N. Kawamura, P. Strasser, T. Nagatomo, K. Shimomura, Y. Miyake, M. Kita, A. Shinohara, K. M. Kubo, T. Miura, J. Radioanal. Nucl. 
Chem. 319, 767 (2019).

(28) G. Yoshida, K. Ninomiya, M. Inagaki, W. Higemoto, P. Strasser, N. Kawamura, K. Shimomura, Y. Miyake, T. Miura, M. K. Kubo, A. Shinohara, J. Radioanal. Nucl. Chem. 320, 283 (2019).

(29) K. Ninomiya, M. Kitanaka, A. Shinohara, M. Tampo, Y, Miyake, Y, Sakai, M. K. Kubo, J. Radioanal. Nucl. Chem. 316, 1107 (2018).

(30) M. Inagaki, K. Ninomiya, G. Yoshida, W. Higemoto, N. Kawamura, Y. Miyake, T. Miura, A. Shinohara, J. Nucl. Radiochem. Sci. 18, 5 (2018).

(31) K. Ninomiya, M. K. Kubo, P. Strasser, A. Shinohara, M. Tampo, N, Kawamura, Y. Miyake, JPS Conf. Proc 21, 011043 (2018).

(32) T. Osawa, K. Ninomiya, G. Yoshida, M. Inagaki, M. K. Kubo, N. Kawamura, Y. Miyake, JPS Conf. Proc. 8, 025003 (2015).

(33) I. Umegaki, Y. Higuchi, H. Nozaki, K. Ninomiya, M. K. Kubo, M. Tampo, K. Hamada, S. Doiuchi, P. Strasser, N. Kawamura, Y. Miyake, J. Sugiyama, JPS Conf. Proc 21, 011041 (2018).

(34) H. Daniel, F. J. Hartmann, R. A. Naumann, Phys. Rev. A 59, 3343 (1999).

(35) K. Ninomiya, T. Nagatomo, M. K. Kubo, P. Strasser, N. Kawamura, K. Shimomura, Y. Miyake, T. Saito, W. Higemoto, J. Phys. Conf. Ser. 225, 012040 (2010).

(36) M. Ueda, I. Taguchi, T. Saito, Institute for Monetary and Economic Studies, 1996, Discussion Paper 1996-E-26.

(37) D. Kessler, H. Mes, A. C. Thompson, H. L. Anderson, M. S. Dixit, C. K. Hargrove, R. J. McKee, Phys Rev. C 11, 1719 (1975).

(38) G. Fricke, C. Bernhardt, K. Heilig, L. A. Schaller, L. Schellenberg, E. B. Shera, C. W. De Jager, Atom. Data Nucl. Data. 60, 177 (1995).

(39) R. Pohl, A. Antognini, F. Nez, F. D. Amaro, F. Biraben, J.
M. R. Cardoso, D. S. Covita, A. Dax, S. Dhawan, L. M. P. Fernandes, A. Giesen, T. Graf, T. W. Hänsch, P. Indelicato, L. Julien, C. Y. Kao, P. Knowles, E. O. L. Bigot, Y. W. Liu, J. A. M. Lopes, L. Ludhova, C. M. B. Monteiro, F. Mulhauser, T. Nebel, P. Rabinowitz, J. M. dos Santos, L. A. Schaller, K. Schuhmann, C. Schwob, D. Taqqu, J. F. C. A Veloso, F. Kottmann, Nature 466, 213 (2010).

(40) K. Ninomiya, T. Kudo, P. Strasser, K. Terada, Y. Kawai, M. Tampo, Y. Miyake, A. Shinohara, M. K. Kubo, J. Radioanal. Nucl. Chem. 320, 801 (2019).

(41) M. Katsuragawa, M. Tampo, K. Hamada, A. Harayama, Y. Miyake, S. Oshita, G. Sato, T. Takahashi, S. Takeda, S. Watanabe, G. Yabu, Nucl. Instr. Meth. Phys. Res. A, 912, 140 (2018)

(42) A. Hillier, K. Ishida, P. Seller, M. C. Veale, M. D. Wilson, JPS Conf. Proc. 21, 011042 (2018).

(43) T. Takahashi, B. Paul, K. Hirose, C. Matsumoto, R. Ohno, T. Ozaki, K. Mori, Y. Tomita, Nucl. Instr. Meth. Phys. Res. A 436, 111 (1999).

(44) H. J. Evans, Nucl. Phys. A 207, 379 (1973).

(45) H. Ejiri, I. H. Hashim, Y. Hino, Y. Kuno, Y. Matsumoto, K. Ninomiya, H. Sakamoto, A. Sato, T. Shima, A. Shinohara, K. Takahisa, N. H. Tran, J Phys Soc Jpn. 82, 044202 (2013).

(46) I. H. Hashim, H. Ejiri, T. Shima, K. Takahisa, A. Sato, Y. Kuno, K. Ninomiya, N. Kawamura, Y. Miyake, Phys Rev C. 97, 014617 (2018).

(47) K. Ninomiya, M. Inagaki, M. K. Kubo, T. Nagatomo, W. Higemoto, N. Kawamura, P. Strasser, K. Shimomura, Y. Miyake, S. Sakamoto, A. Shinohara, T. Saito, J. Radioanal. Nucl. Chem. 309, 65 (2016).

(48) K. Ninomiya, M. K. Kubo, P. Strasser, T. Nagatomo, Y. Kobayashi, K. Ishida, W. Higemoto, N. Kawamura, K. Shimomura, Y. Miyake, T. Suzuki, A. Shinohara, T. Saito, JPS Conf. Proc. 8, 033005 (2015). 